\title{
Hemostasis in the Lab, Approaching to the Correct Diagnosis in the Coagulopathies
}

\author{
Lopez $S \mathbf{N}^{1}$, Cortina DLR E $\mathbf{E}^{2}$, Gonzalez $\mathbf{P ~ M}^{1}$, Nunez $T M^{1, *}$ \\ ${ }^{1}$ Pediatric Hematologists, Hematology Department, National Institute of Pediatrics, Mexico City, Mexico \\ ${ }^{2}$ Laboratory of Thrombosis, Fibrinolysis and Platelet Function, National Institute of Cardiology, Ignacio Chavez, Mexico City, Mexico
}

\author{
Email address: \\ marantoscano@gmail.com (Nunez T M) \\ ${ }^{*}$ Corresponding author
}

\section{To cite this article:}

Lopez S N, Cortina DLR E, Gonzalez P M., Nunez T M. Hemostasis in the Lab, Approaching to the Correct Diagnosis in the

Coagulopathies. American Journal of Pediatrics. Vol. 6, No. 2, 2020, pp. 98-104. doi: 10.11648/j.ajp.20200602.16

Received: October 2, 2020; Accepted: February 24, 2020; Published: March 6, 2020

\begin{abstract}
Coagulation is the result of a coordinated interaction of blood proteins, circulating cells, endothelium cells and extracellular matrix proteins in the vessel wall. This process works in conjunction with other mechanisms that have to keep the blood in an effective flow, without hemorrity when damage occurs or without a thrombus when clotting begins. Most of the time, there is not so much information that a clotting test gives us, however, in the face of a real clinical scenario that concurrent with a personal haemorrhage, this is essential to take action. In addition, there is a thrombophilic scenario that has to be investigated. In all of them, it is important to note that doctors have to take into account that the pre-analytical phase plays a fundamental role, so it is very important to look around any situation that may impact the patient's results. There is a wide range of laboratory tests to evaluate different mechanisms of hemostasis, according to the history and characteristic of the clinical patient. With this in mind, there is a right time to ask for the right test, to get a better result for diagnosis and treatment. Analytical quality is the set of actions that are carried out in the laboratory with the aim of obtaining an accurate result, reflecting the real situation of the patient. Grossly, platelets, clotting factors and the fibrinolytic system could be studied, not all patients need all tests, they could be solved with a simple test, but may require a deep focus; that is why the patient with a history of mucocutaneous bleeding has to be studied for thrombocytopaties or VWD; a patient with muscle or joint bleeding has to be a factor deficiency test. We propose an initial approach to detecting the pathological situations essential to make decisions. Each person has different symptoms and different approaches, which is why identification and the right tests give us the greatest opportunity to get the right diagnosis.
\end{abstract}

Keywords: Blood Coagulation, Hemostasis, Blood Test, Clinical Laboratory, Coagulopathty

\section{Introduction}

\subsection{Initial Approach}

Coagulation [1] is the result of a coordinated interaction of blood proteins, circulating cells, cells of the vasculature and proteins of the extracellular matrix in the vessel wall. This complex mechanism makes it difficult to evaluate it in the laboratory, which only restricts itself to measuring the coagulation proteins and circulating cells, while the vascular elements are not measurable.

When is necessary study a patient with abnormal coagulation test, the preanalytical phase plays a fundamental role, if this takes wrong way, the results will not be assessable, affecting the efforts from hemostasis laboratory team that are aimed providing excellence in care, which means reporting an accurate results, in the shortest time and the lowest possible cost according with the International standard care.

\subsection{Preanalytical Phase}

The preanalytical phase involves all those factors that affect the quality of the sample (identification, patient condition, adecuate sampling collection, correct conservation and transport of the sample until it will be used). 
The analytical quality is the set of measurements that are carried out in the laboratory with the aim of obtaining an accurate and precise result. [2]

Currently, laboratory tests that are available to evaluate different phase, depends on the sintomatology, is the test that should be order.

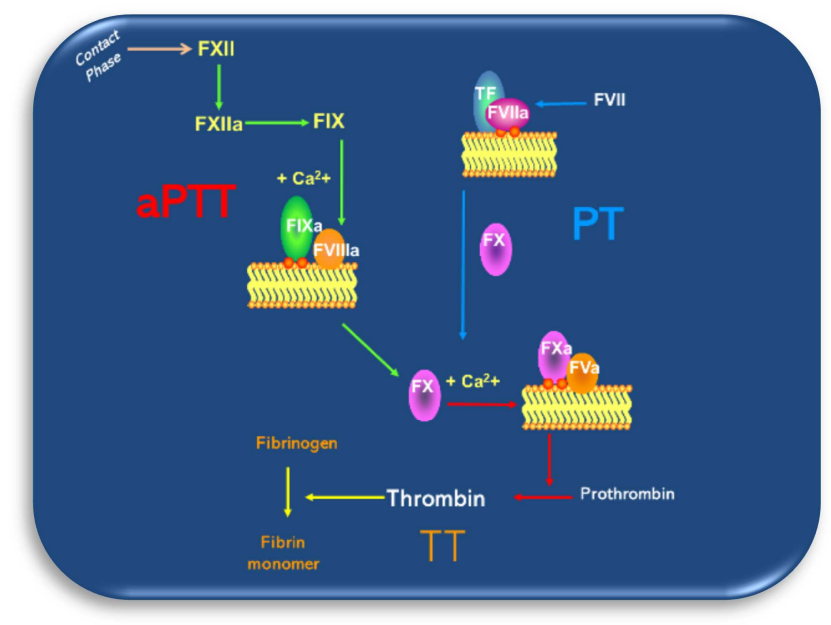

Figure 1. Shows the coagulation pathways.

aPTT: activated partial thromboplastin time, PT: prothrombin time, TT: Thrombin time.

\section{The Correct Approach}

\subsection{The Platelet Phase; The Beginning}

The platelet disorders symptoms are characterized by mucocutaneous bleeding; The principles causes of thrombocitopenya is show in Figure 2.

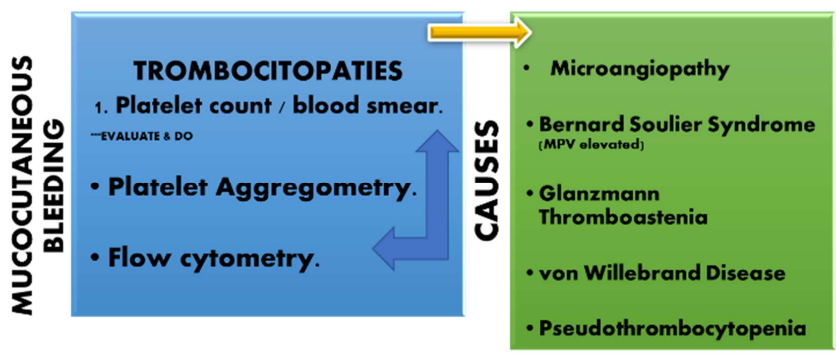

Figure 2. Study a patient with mucocutaneous hemorrhage.

For the platelet study used in the past; the bleeding time, by the Ivy's or Mielke's technique. Bleeding time (BT) was a global test of primary hemostasis, its greatest utility was evaluate the platelets function; it can be altered in certain circumstances such as uremia, the use of some drugs such as antiplatelets, non-steroidal anti-inflammatory drugs (NSAIDs), ticlopidine among others, the same happens with low platelet counts, likewise in the alteration of platelet glycoproteins as in Glanzmann thrombasthenia, or von Willebrand's disease, but this test has not enough sensibility/specificity, so its use is not recommended actually. [3] Have fallen into disuse, due to the difficulty in its standardization.

\subsection{Platelet Evaluation \& Thrombocytopathies}

To evaluate the platelet function, exist different tests but it is essential first of all, obtained the platelet count and the platelet size by CBC and the volume platelet mean (VPM), normally goes from 5-12 femtoliters (fL), and a normal count goes between 150 - 450,000 cells / $\mu \mathrm{L}$.

Thrombocytopenia may be secondary to an increase in peripheral blood destruction, which is often associated with increased VPM, while lack of production in bone marrow $(\mathrm{BM})$ is associated with decreased VPM. The decrease in platelets number can be the consequence of an antibody sensitive to EDTA that promotes the agglutination of platelets, known as pseudothrombocytopenia.

The most frequent cause of elevation in the number of circulating platelets is iron deficiency, followed by some other reactive causes such as infection, however, if thrombocytosis persists for more than 6 months, the possibility of a myeloproliferative disorder should be considered.

In the past, the clot retraction test was widely used [4], the test has the basis that the retraction of the clot is produced through the interaction of glycoprotein $\alpha \mathrm{IIb} \beta 3$ (GIIb-IIIa) with the actin of the cytoskeleton, a process that takes the rearrangement of the platelet membrane. Retraction requires ATP as an energy source.

It depends on several factors:

a) Quantity and quality of the platelet,

b) Fibrinogen concentration,

c) Hematocrite.

This test has low sensitive and its influenced by the cleaning of the material [1] is not recommended.

The test that offers a good sensitivity and specificity to assess platelet defects is platelet aggregometry, described by Borhn in 1962, which has been revised and modified at the present [5]. It consists of measuring in real time the aggregation of platelets in a sample of platelet-rich plasma (PRP) by optical clearance. It requires a spectrophotometer in which the PRP sample is deposited in an incubation cuvette at $37^{\circ} \mathrm{C}$, which is between a light source and a photocell that calculates the optical density or turbidity of the PRP. Several substances known for their agonist effect on platelets are added, in order to induce aggregation of platelets and simulate in vitro what happens in vivo. However, it must be remembered that during the neonatal period it can be a confuse test since it has been shown that neonatal platelets normally respond less to agonists, by the other side we have to considere is necessary a great blood volume. [6] So the flow cytometry would be much more helpful during this period. [7]

Something that we must have to considerer in the first instance for the aggregometry, is the platelet count, since in patients with thrombocytopenia the reported values will not be accurate, so international recommendation is a platelet count at least over $100 \times 10^{9}$ cells $/ \mathrm{L}$.

Like all the rest of coagulation test, the preanalytical phase it's crucial, some food and drugs can affect platelet function; 
as garlic, chocolate, different kind of oils, alcohol, onions, peppers, ginger, NSAIDs, antibiotics, including antacids, so we must be cautious, for an accurate result.

The most commonly used agonist agents are ADP, epinephrine, collagen, ristocetin and arachidonic acid. The aggregation cinetica interpretation should always be done by an hematologist trained.

In Figure 3 the normal aggregation pattern is shown.

If a pattern of abnormal aggregation is observed in a patient, it is advisable to repeat the evaluation at least once, to verify that the abnormality persists, since the collection time, the centrifugation time, the platelet count, the $\mathrm{pH}$, mixing speed, hematocrite, temperature, lipemia, air bubbles among others factors can interfere with the results.

The test helps us to diagnoses some thrombocitopaties like: Bernard Soulier disease, Glanzmann thromboastenia, some types of von Willebrand Disease (vWD), as well as cyclooxygenase alterations, and in patients with antiplatelet drugs it helps us to monitor their use and stablish resistance.

Flow cytometry [8] is the most accurate, quick, objective and quantitative method to study platelet membrane glycoproteins, the expression of some glycoproteins, like IbIX and $\mathrm{V}$ is diminished, it consists in the evaluation of the constitutive glycoproteins of the platelet membrane using markers such as CD41, CD42, CD61, CD62; as we describes:

CD41: recognises the platelet membrane glycoprotein GPIIb (the integrin alpha IIb chain) which is non-covalently associated with GpIIIa (the integrin beta 3 chain) to form the GpIIb/IIIa complex.

CD61: recognises the platelet membrane glycoprotein GpIIIa (the integrin beta 3 chain).

CD42b: reacts with GPIb on megakaryocytes and platelets. $\mathrm{CD} 42 \mathrm{~b}$ also inhibits ristocetin-dependent binding of Von Willebrand Factor to platelets and ristocetin-induced platelet agglutination [8].

CD62p: P-selectin is found in the $\alpha$-granules of platelets. Presence of CD42 on the platelet surface indicates platelet activation, while their abscense could be implicated a granules disease. [9]

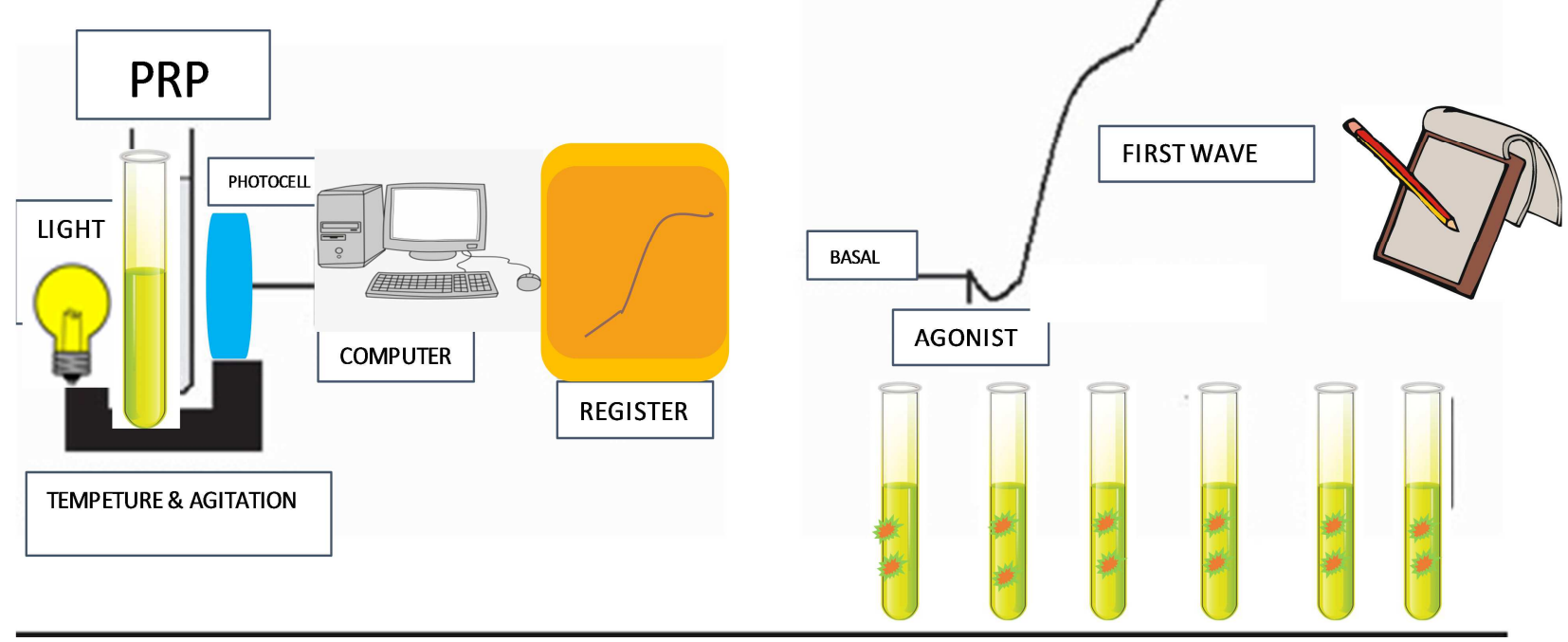

Figure 3. Process \& normal platelet aggregation curve.

\subsection{Point of Care Test [10]}

Point of Care tests, such as the platelet function test (PFA100) is not considered as a screening test for platelet dysfunction, it was originally thought for $\mathrm{vWD}$, however emerge as an alternative for the monitoring of the various antiplatelet agents, other tests as point of care can be mention are: [11].

\subsubsection{The Impact Cone and Plate (let) Analyzer}

Is shear-induced platelet adhesion/aggregation onto surface in whole blood. Is use for screening of congenital primary hemostasis abnormalitieswith low sensitivity ans specificity; as well for evaluation of platelet response to aspirin and clopidogrel.

\subsubsection{The VerifyNow System}

Is due Fibrinogen-platelet agglutination in response to agonist in whole blood, to monitoring of the platelet response to antiplatelet agents.

\subsubsection{The Plateletworks}

Is due platelet counting pre- and post-activation in whole blood, to monitoring of the platelet response to antiplatelet agents.

However the results are not encouraging and these test are not recommended as a routine for the diagnosis of congenital thrombocitopthies, very useful for monitoring antiplatelet agents. [12] 


\section{Coagulation Factors, the Second Phase}

On the other hand coagulation tests such as: prothrombin time (PT) and activated partial thromboplastin time (aPTT) are used as screening tests to evaluate the coagulation pathways, and hemorrhage risk, traditionally describes, intrinsic and extrinsic pathway of coagulation in a didactic way, however this is explained better in the coagulation cellular theory. [13]

The factors involved in the intrinsic pathway of coagulation are evaluated by the aPTT, while the PT evaluates the extrinsic pathway, both coincide in the common pathway (Figure 1).

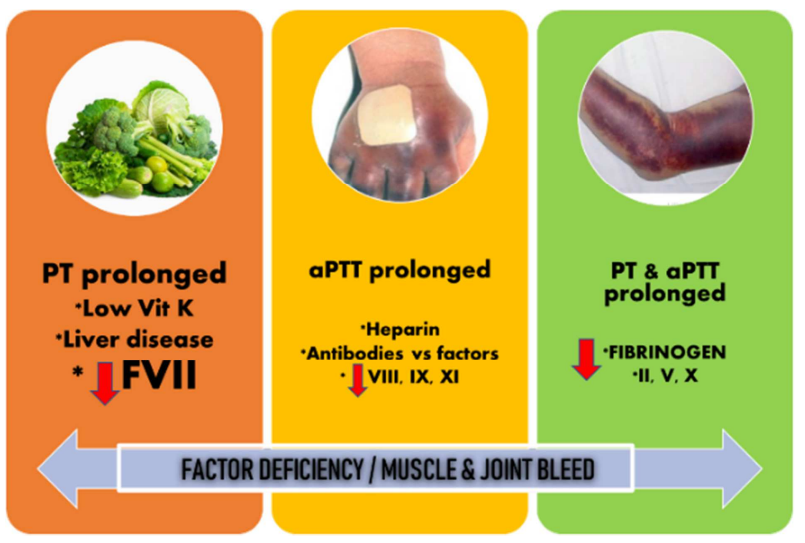

Figure 4. $P T \&$ aPTT Approach.

Characteristically, patients with factor deficiency present joint or muscle hemorrhages. Figure 4, shows a quick review for factor defects.

\subsection{The Blood Sample}

Blood samples for coagulation tests, should be drawn into siliconized sodium citrated tubes in a 9:1 (total blood: anticoagulant relation), the tubes must be filled up to $90 \%$ but no more than $110 \%$, it is important that if the amount of anticoagulant is inappropriate, because higher citrate concentrations may lead to longer clotting times, results that confuse the clinician, the amount of citrate interferes with the $\mathrm{Ca}^{2+}$, used during the test, this mistake has been significantly reduced with the vacuum tubes with negative pressure currently available, since they are calibrated to extract the exact amount of blood to maintain the proper proportion with the anticoagulant. However, an error that is frequently observed is in patients who have high hematocrite ( $>55 \%)$ and that consequently their plasmacrite is diminished and therefore the aforementioned blood / citrate relationship is not respected, so that will have to adjust the amount of citrate with which the sample is conserved, the formula for its correction is the following:

The patient's Hematocrite is substituted in the following formula:

Anticoagulant volume $(\mathrm{mL})=1.85 \times 103(100-$ Hto patient $) *$ total blood volume $(\mathrm{mL})$.

Until now there is not enough evidence to adjust in the citrated tube in patients with severe anemia.
Another important aspect is the time elapsed between the blood collection and the performance of the tests, since, if more than 4 hours elapse, some factors such as FV and FVII are inactivated, results in prolonged coagulation times.

\subsection{Screening Coagulation Tests}

\subsubsection{Prothrombin Time (PT)}

In the prothrombine time the clot formation results from the FVII activation with tissue factor (thromboplastin) and $\mathrm{Ca}^{2+}$, the normal result is $10-14$ seconds, it is important to remember the reference values of each laboratory and the age of the patients.

The result of the prothrombin time can be expressed in seconds, percentages $(\%)$ or in ratios.

The report is internationally recommended in seconds and the reason depend strongly on the reagent and the instrument used; to report in (\%) a calibration curve should be made with a pool of normal plasmas or a commercial calibrator [14]. Current recommendations express their concern to continue using the percentage of activity given that it does not have a linear relationship and can fall into interpretation errors.

Depending on the type of thromboplastin (human placenta, rabbit brain, mouse or monkey and currently the recombinant) that the result is added can vary widely, so a standardized method has been developed to express these variations: International Normalized Ratio (INR), the importance of this parameter lies in its usefulness to evaluate the effectiveness of anticoagulation with vitamin $\mathrm{K}$ antagonists, but it has little use in other conditions of coagulopathy such as liver failure.

$$
\left(\left[\mathrm{PT}_{\text {pat }} / \mathrm{TP}_{\mathrm{NGM}}\right]^{\mathrm{ISI}}=\mathrm{INR}\right.
$$

Therefore, it is suggested internationally that INR should only be reported for patients using vitamin $\mathrm{K}$ antagonists (VKA), currently there is a capillary TP which facilitates the monitoring of patients and their use with VKA.

Some causes of PT prolonged are hepatopathy, vitamin $\mathrm{K}$ deficiency, factor deficiency VII.

\subsubsection{Activated Partial Thromboplastin Time (aPTT)}

The aPTT evaluates the intrinsic pathway of coagulation. For this reaction to the citrated plasma, phospholipids, and a contact factor initiator such as kaolin, silica, celite or ellagic acid are added, then the incubation and finally $\mathrm{Ca}^{2+}$ is added. Thus, the sensitivity of the reagent depends on the origin, concentration of phospholipids, type of activator, ionic strength and stabilizers.

The normal result varies from 25-45 seconds; However, it is important to remember the reference values of each laboratory and the age of the patients.

The most frequent cause of the alteration of aPTT is the deficiency of one of the factors of the intrinsic pathway (II, V, VIII, IX, XI or XII), although this must be a place with an activity $<50 \%$ to modify it, the most frequent cause is the use of unfractionated heparin (UFH) when the blood sample is taken through the catheters that are heparinized even with many people and after "washing" the catheter, we may have falsely prolonged aPTT; however, it is recommended internationally for the monitoring of UFH [15], as well as the 
presence of acquired inhibitors such as aPTT, and the most frequent of these is the lupus anticoagulant, one way to differentiate them is by mixing with normal plasma, this is done with a volume of the patient's plasma plus a normal plasma volume, the correction should be observed in the first dilution since we remember that we only need $50 \%$ of the concentration of factors to present a normal coagulation and this directs the clinician to look for a deficiency of the factors of this way, this correction must be valued by the Rosner index, which is calculated in the following way:

$$
((\text { aPTT mix }+ \text { aPTT pool }) / \text { aPTT patient }) * 100
$$

Where each laboratory should establish the correction values, and if don't get the correction index, considered an inhibitor.

Is a different history for the patient with thrombosis, the Figure 5 shows the approach of prolonged aPTT \& thrombosis, where a inhibitor could be present, or a contact factor low level as Factor XII [16] has to be test.

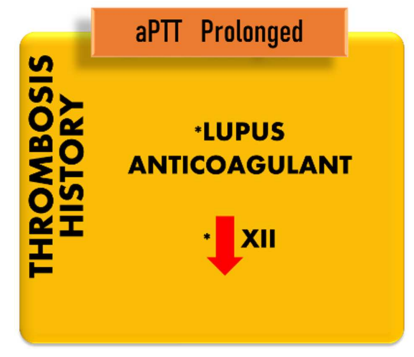

Figure 5. aPTT \& Thrombosis.

\subsubsection{Thrombin Time (TT)}

The thrombin time allows to evaluate the fibrin formation stage, by measuring the time it takes to coagulate the citrated plasma in the presence of thrombin, its reference value is between 15 to $20 \mathrm{sec}$. The reference values vary with the concentration of thrombin used. Thrombin time is prolonged with low levels of fibrinogen or dysfibrinogenemia, treatment with UFH (however, it is not used for monitoring), high levels of fibrin digestion products (FDP), the presence of paraproteins, as well as inhibitors of thrombin. [17]

Another screening test is reptilase time (RT). It is a coagulation time like the thrombin time, but where the clot is produced by the action of an enzyme of viper venom called reptilase. Reptilase transforms fibrinogen. It differs from the thrombin action on fibrinogen in that it releases only fibrinopeptide A. Reptilase time is not inhibited by heparin and can be used in place of the thrombin time in the evaluation of fibrinogen in heparinized patients. When evaluating hypercoagulability states, a normal result of the reptilase time excludes the presence of abnormal fibrinogen. If the effect of heparin is the only cause of prolonged prothrombin time, the RT will be normal.

The simultaneous alteration of PT and aPTT frequently indicates an alteration of both the intrinsic and extrinsic pathways, such as the case of liver disease, coagulopathy due to consumption, in less frequent cases. (Figure 1). Under these conditions, a utility will be obtained to measure FDP, D Dimer (DD) and fibrinogen levels that allows to orient the origin of a simultaneous alteration, of course in the correlation with the clinic manifestations.

\section{Fibrinogen, the Sticky Phase}

The fibrinogen [18], the last protein of the coagulation cascade, can be measured by chemical or immunological methods, and is found in concentrations ranging from 200$400 \mathrm{mg} / \mathrm{dL}$.

In fibrinogen deficiency o dysfibrinogenaemia, patients traditionally show muco-cutaneous hemorrhages.

The fibrinogen level can increase up to $800 \mathrm{mg} / \mathrm{dL}$ in acute inflammatory process.

\subsection{Measurement}

Currently, its measurement is recommended by the Clauss method, which is functional and is based on the clottrable fibrinogen in the presence of thrombin excess. The fibrinogen concentration in $\mathrm{g} / \mathrm{L}$ is obtained by comparing the clotting time of the plasma sample with serial dilutions of a reference plasma with known fibrinogen concentration. [19]

As it is a semiautomatic coagulometric method, half of the indicated volumes can be used, this decreases the amount of sample needed, the presence of heparin or high levels of FDP give falsely diminished results.

\subsection{Breaking Bad}

D-dimer is a soluble fibrin digestion product from the plasmin action on fibrin formed, thus is a coagulation and fibrinolytic marker.

The measurement of DD [20] that is carried out by means of a specific monoclonal antibody against the $\mathrm{D}$ regions of fragmented fibrin, is a biomarker of fibrin formation, which not only rises in thrombosis, it is normally elevated in pregnancy, inflammatory processes, neoplastic, with age and others. It is more specific and sensitive test in patients with DIC, wich values arise over than $500 \mathrm{ng} / \mathrm{mL}$.

Remember only values below 50mg of fibrinogen alter PT/aPTT.

\section{Viscoelastic Test, the Vintage Era}

There is an assay modality in the patient with coagulopathy, the so-called viscoelastic tests such as thromboelastography (TEG) and thromboelastometry (ROTEM) which describe in a graph, the global interaction of different components of the hemostatic system, such as coagulation factors, fibrinogen, platelets and fibrinolytic system, since whole blood is used and the kinetic and viscoelastic characteristics of the clot are evaluated in real time. [21]

Heparinase to deactivate heparin is now employed in the TEG (r) technology to prevent interference from heparin in samples. 
Which allow us to evaluate hemostasis in a global way and allows us to make decisions immediately in the critical patient, just what the patient need, low risk better results.

Nomenclature Differences for ROTEM. [22]

Although TEG and ROTEM measure the same coagulation functions there is a different naming convention for each device.

\subsection{Features of a Teg Curve}

\subsubsection{Initiation (R)}

Represents period of time of latency from start to initial fibrin formation due to effects of Factor VIIa and Tissue Factor.

\subsubsection{Amplification (K)}

Represents time taken to achieve a certain level of clot strength due to thrombin and activation of platelets (where rtime $=$ time zero)

\subsubsection{Propagation ( $\alpha$-Angle)}

Measures the speed at which fibrin build-up and crosslinking takes place (clot strengthening), and hence assesses the rate of clot formation.

\subsubsection{Maximum Amplitude (MA)}

A function of the maximum dynamic properties of fibrin and platelet bonding via GPIIb/IIIa and represents the strongest point of fibrin clot and correlates to platelet function: $80 \%$ platelets; $20 \%$ fibrinogen.

\subsubsection{Clot Stability (LY 30\%)}

This is percentage decrease in amplitude 30 minutes postMA and gives measure of degree of fibrinolysis.

In Figure 6, shows an example of the curve that is generated in a patient without pathology.

\subsection{Rotem}

ROTEM offers some advantages as portable device, and dinamyc test, for the platelet, factors and fibrinogen; more accurate viscoelastic test.

But both are incredible useful in the critical bleeding patient.

A normal TEG/ROTEM shows in Figure 6.

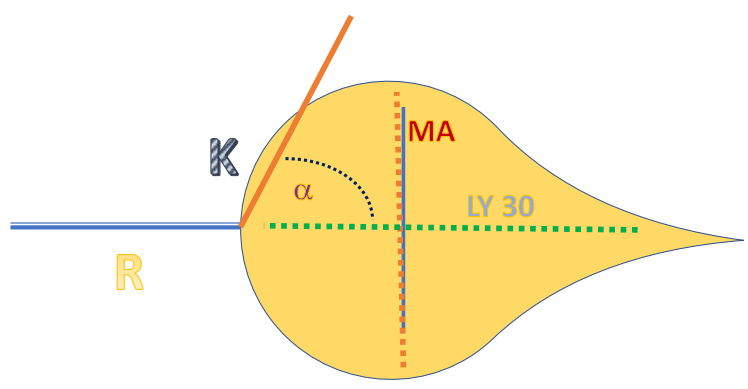

Figure 6. Normal teg curve.

\section{Conclusions}

Once an adequate clinical evaluation of the patient with hemorrhage has been carried out, we will be able to guide the type of studies to be requested, which will also finally allow us to define the treatment to be followed according to the proposed approaches in this review, that is a quick form to evaluate a patient, depending of the symptoms and the correct test in the correct time to get a better diagnosis and treatment for our patients.

\section{References}

[1] Izaguirre R, De Micheli A. 2005. Evolución del conocimiento sobre la sangre y su movimiento. Parte II. El saber sobre su composición. Iatroquímica de la sangre. Rev Invest Clin.; 57: $85-97$

[2] Kordich L. 2006. Fundamentos para el manejo practico en el laboratorio de Hemostasia. Grupo CATH.

[3] Zamora-González. 2012. Pruebas del coagulograma y componentes de la hemostasia. Utilidad para diagnosticar las diátesis hemorrágicas Revista Cubana Hematología, Inmunología y Hemoterapia; 28 (2): 141-150.

[4] Zimring JC. 2009. Introduction to coagulation testing. Transfusion Medicine and hemostasis, Clinical and laboratory aspects. Chirstopher D Hillyer, Beth H Shaz, James C Zimring, Thomas C Abshire Editors, Elsevier, New York USA., pp|601-605.

[5] Jae-Lim Choi, Shuhua Li, and Jin-Yeong Han. 2014. Platelet Function Tests: A Review of Progresses in Clinical Application. BioMed Research International Volume, Article ID 456569, 7 pages.

[6] Martha Sola-Visne. 2012. Platelets in the neonatal period: developmental differences in platelet production, function, and hemostasis and the potential impact of therapies. ASH, Hematology; 506-511.

[7] Baker-Groberg S, Lattimore S. 2016. Assessment of neonatal platelet adhesion, activation, and aggregation., et al J Thromb Haemost. April; 14 (4): 815-827.

[8] Michelson, A. D., 2006. Evaluation of platelet function by flow cytometry. Pathophysiol Haemost Thromb,. 35 (1-2): p. 67-82.

[9] Malays J Med Sci. 2013; 20 (2): 62-66. New Horizons in Platelets Flow Cytometry. Malays J Med Sciv. 20 (2).

[10] Elisabeth Mahla. 2009. Point-of-Care Platelet Function Analysis. Journal of the American College of Cardiology. Vol. 53, No. 10.

[11] Paniccia R, Priora R, Alessandrello Liotta A, Maggini N, Abbate. 2014. Assessment of platelet function: Laboratory and point-ofcare Methods. World J Transl Med August 12; 3 (2): 69-83.

[12] Oh Joo Kweon, Yong Kwan Lim, Bohyun Kim, et al. 2019. Effectiveness of Platelet Function Analyzer-100 for Laboratory Detection of Anti-Platelet Drug-Induced Platelet Dysfunction. Ann Lab Med; 39: 23-30 https://doi.org/10.3343/alm.2019.39.1.23.

[13] Hoffman M. Remodeling the blood coagulation cascade. 2003. J Thromb Thrombolysis. 16 (1-2): 17-20. 
[14] Tripodi A, Lippi G, Plebani M. 2016. How to report results of prothrombin and activated partial thromboplastin times. Clin Chem Lab Med; 54 (2): 215-222.

[15] Byun JH, Jang IS, Kim JW, Koh EH. 2016. Stablishing the heparin therapeutic range using aPTT and anti-Xa measurements for monitoring unfractionated heparin therapy. Blood Res; 51: 171-4.

[16] Evi Stavrou and Alvin H. Schmaier. 2010. Factor XII: What Does It Contribute To Our Understanding Of The Physiology and Pathophysiology of Hemostasis \& Thrombosis. Thromb Res.; 125 (3): 210-215. doi: 10.1016/j.thromres.2009.11.028.

[17] Kitchen S, Geisen U, Kappelmayer J, Quehenberger P, et al. 2018. Evaluating the analytical performance of four new coagulation assays for the measurement of fibrinogen, Ddimer and thrombin time. Int J Lab Hematol; 40: 637-644.

[18] Jennings I, Kitchen DP, Woods TA, Kitchen S, Walker ID. 2009. Differences between multifibrin $U$ and conventional Clauss fibrinogen assays: data from the UK National External
Quality Assessment scheme. Blood Coagul Fibrinolysis; 20: 388-90.

[19] Lowe G, Rumbley A, Mackie IA. 2004. Review Article. Plasma Fibrinogen. Ann clin Biochem; 41: 430-440.

[20] Olson JD et al. 2015. D-dimer: An Overview of Hemostasis and Fibrinolysis, Assays, and Clinical Applications. Adv Clin Chem. Volume 69, Pages 1-46.

[21] López MS. 2018. Tromboelastografía y tromboelastometría para el monitoreo del tratamiento en pacientes con sangrado en distintos escenarios clínicos. HEMATOLOGÍA, Volumen 22 Número Extraordinario, XIII Congreso del Grupo CAHT: 278-291.

[22] Milind Thakur, Aamer B Ahmed. 2012. A Review of Thromboelastography. International Journal of Perioperative Ultrasound and Applied Technologies. 1 (1): 25-29. 